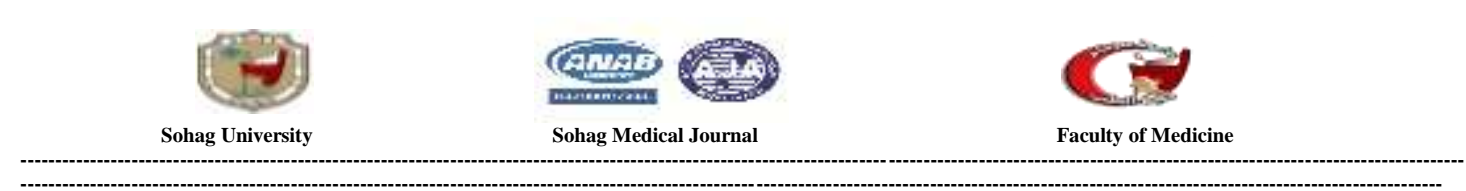

\title{
Clinico-laboratory profile in children with status epilepticus and correlation to short-term outcomes at Sohag University Hospital
}

\author{
Asmaa mansour, Abdelrahim Abdrabou Sadek, Ashraf Abou-Taleb \\ Departments of Pediatrics, Faculty of Medicine, Sohag University
}

\begin{abstract}
Background: Status Epilepticus is a major neurological and medical emergency. Despite the advance in treatment, it is still associated with mortality and morbidity. It is a common pediatric neurological emergency with an estimated incidence of 18-23 per 100,000 children per year and mortality of $2 \%-7 \%$.

Objective: The objectives of the study were to determine the clinical profile, immediate outcome and possible risk factors of SE in the pediatric age group admitted at Sohag University Hospital.

Patients and Methods: In this research, we have studied 100 patients who were admitted to the pediatric department over a period of 1 year 56 were males and 44 were females, Their ages ranged from one month to 15 years with a mean age was $37.8 \pm 44.4$ months and those patients were subjected to full clinical histories, clinical and laboratory examination.

Results: In our study, we found that $38 \%$ of patients were improved without any neurological deficits.

$15.8 \%$ of patients who had epileptic seizures were related significantly to improved outcomes, while recurrence outcome represents about $59.3 \%$ in those patients who presented with epilepsy. patients presented with hypoxic-ischemic encephalopathy show the highest mortality rate $(38.1 \%)$ among the deaths( $21 \%)$.

Conclusion: In this study, we found that in the included children, epilepsy is the most common cause of status epilpticus and those epileptic children were significantly related to the improvement and recurrence outcome, patients presented with hypoxicischemic encephalopathy were significantly related to death outcome and all patients presented with status epilepticus should have full clinical examination and laboratory investigations.
\end{abstract}

Keywords: Clinico-laboratory, Children, Status epilepticus

\section{Introduction}

Status epilepticus (SE) is considered to be a significant neurological crisis. Regardless of the treatment advances, it is still accompanied by huge morbidity and mortality [1].

The status epilepticus conventional meaning defined as 'Consistent seizure action going on for 30 minutes or more, or irregular seizure movement going on for over a short ways from which patient doesn't recapture cognizance, it is ending up progressively perceived that seizure term of beyond what 10 minutes can prompt cerebrum harm and length of seizure action in meaning of status epilepticus is being diminished [2].

The International League Against Epilepsy [3], defines status epilepticus as "a condition resulting either from the failure of the mechanisms responsible for seizure termination or from the initiation of mechanisms which lead to abnormally prolonged seizures(after time point $\mathrm{t} 1$ ), that first time point indicates the earliest time 
when treatment should be started. It is a condition that can have long-term consequences(after time point $\mathrm{t} 2$ ), including neuronal death, neuronal injury, and alteration of neuronal networks, depending on the type and duration of seizures".

It is estimated that 1.3 to $16 \%$ of all patients with epilepsy will develop SE at some point in their lives [4].

Approximately $70 \%$ of SE occurs in children less than one year, $75 \%$ in less than three years of age and the first episode most commonly occurs around 2.5 years after initial diagnosis [5].

It has been observed that $20 \%$ of individuals with epilepsy will have an episode of SE within 5 years of the initial diagnosis [6].

\section{Aim of the work}

The objectives of the study were to determine the clinical profile, immediate outcome and possible risk factors of SE in the pediatric age group admitted at Sohag University Hospital.

\section{Patients and Methods}

1-Study design: It is a prospective hospitalbased study.

2- Setting: The study was conducted in pediatric intensive \& intermediate care and pediatric emergency room units at Sohag University Hospital.

The study was conducted from 1 December 2016 to 30 November 2017.

3- Study protocol: Patients who referred to the pediatric department in Sohag University Hospital and who presented with status epilepticus were included in the study.

4- Study population: This study included 100 patients who presented with status epilepticus during their stay at the pediatric department at sohag university hospital and their ages ranging from 1 month to 15 years.

5-Ethical consideration: was approved

6- Patient handling \&data collection:

All patients in this study were subjected to the following:

\section{(A) Clinical histories focusing on:}

- Age.

- Gender.

- Past history of SE and seizures.

-Etiology of SE.

- Developmental history.

- Family history of seizures.
- Duration of SE.

- Seizure types.

- Medical treatment in the acute period of SE.

- Sequelae after SE, and the degree of sequelae, if any.

(B) Complete clinical examinations focusing on:

- The general examination includes vital signs, Anthropometric measurements.

- The systemic examination includes Head and Neck examination (fontanel, Neck stiffness), Chest examination, Cardiac examination, abdominal examination, Back and Genitalia.

- Neurological examination includes Consciousness, Speech, Muscle tone and power, Reflexes and Meningeal signs.

(C) Laboratory data:

- Complete blood count.

- Serum electrolytes.

- Serum urea and creatinine.

- ABG.

- Serum ammonia and lactate.

- Extended metabolic screen.

- C.S.F analysis.

- EEG findings during SE and/or after SE.

- Cranial CT and/or MRI findings after SE.

Statistical analysis: Our study includes

A-Descriptive statistics which are:

- Demographic data of the studied population.

- Clinical examination of the studied population.

- Laboratory and other investigations of the studied population.

- Possible etiologies of status epilepticus.

- Medical treatment during the attack of status epilepticus.

- An immediate outcome of the studied population.

B-Correlation between the immediate outcome of the studied population and clinical features, examination, investigations, and treatment of the included population.

Data were analyzed using STATA version 14.2 (Stata Statistical Software: Release 14.2 College Station, TX: StataCorp LP.). Quantitative data were represented as mean, standard deviation, median, and range. Data were analyzed using student t-test to compare the means of two groups and ANOVA for comparison of the means of three groups or 
SOHAG MEDICAL JOURNAL Clinico-laboratory profile in children with status epilepticus and correlation

more. When the data were not normally distributed Kruskal Wallis test for comparison of three or more groups and the MannWhitney test was used to compare two groups. Qualitative data were presented as number and percentage and compared using either the Chisquare test or the Fisher exact test.

\section{Results}

Our study conducted in 100 patients; 56 males and 44 females who were admitted to the Pediatric department of Sohag University Hospital with status epilipticus. Their ages ranged from one month to 15 years with a mean age of $37.8 \pm 44.4$ months and males predominance $(56 \%)$.

We found that generalized tonic-clonic convulsions represented the most common

type of seizures encountered in the studied population $(48 \%)$ followed by focal convulsions (28\%). The mean GCS was $9.6 \pm 2.8$ and ranged from 5 to 15 . Regarding the mean duration of fits, it was $47.4 \pm 19.0$ minutes with a range from 30 to 120 minutes. The developmental assessment showed that the majority has normal development $(68 \%)$. The past history of SE was defined in 28 cases $(28 \%)$, while a family history of similar condition was defined in 18 cases $(18 \%)$, meanwhile positive consanguinity was detected in 67 cases $(67 \%)$ (Table 1).

\section{Table (1): Demographic and clinical} features

\section{of the studied population}

\begin{tabular}{|l|c|}
\hline Variable & Summary statistics \\
\hline Age/months & $37.8 \pm 44.4$ \\
Mean \pm SD & $18(1.5-168)$ \\
Median (range) & $44(44.0 \%)$ \\
\hline Gender & $56(56.0 \%)$ \\
F & \\
M & $28(28.0 \%)$ \\
\hline Type of convulsion & $48(48.0 \%)$ \\
Focal & $24(24.0 \%)$ \\
GTCC & \\
Mixed seizures type & $9.6 \pm 2.8$ \\
\hline GCS & $9(5-15)$ \\
Mean \pm SD & \\
Median (range) & $47.4 \pm 19.0$ \\
\hline Duration/minutes & $44(30-120)$ \\
Mean \pm SD & \\
Median (range) & \\
\hline Development & \\
\hline
\end{tabular}

\begin{tabular}{|l|c|}
\hline Normal & $68(68.0 \%)$ \\
Delayed & $32(32.0 \%)$ \\
\hline Past history of status & $72(72.0 \%)$ \\
No & $28(28.0 \%)$ \\
Yes & \\
\hline Family history of & $82(82.0 \%)$ \\
similar condition & $18(18.0 \%)$ \\
No & \\
Yes & $33(33.0 \%)$ \\
\hline Consanguinity & $67(67.0 \%)$ \\
Negative & \\
Positive & \\
\hline
\end{tabular}

The correlation between immediate outcome and demographic and clinical characteristics of the included patients revealed that younger ages (mean24.3 \pm 41.5 ) were significantly correlated with death outcome, while older ages $(51.0 \pm 39.8)$ were significantly correlated with discharged outcome and have a neurological deficit(Table2). According to the type of convulsions; focal convulsions were significantly related to death outcome $(52.4 \%)$, and generalized convulsions were significantly related to improved outcomes $(68.4 \%)$ (Table 2). Higher GCS (10.9 \pm 2.9$)$ was significantly related to the recurrent status, while lower GCS $(8.7 \pm 1.9)$ was significantly correlated with improved outcomes (Table 2). Longer the duration of status epilepticus was significantly correlated with death outcome $(52.8 \pm 14.0)$, while the shorter the duration of status epilepticus was significantly related to improved outcome $(45.4 \pm 22.3)$ and recurrence outcome

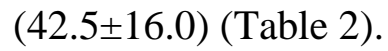

As regard development of the studied population; normal development was significantly related to improved outcome

(79\%) and (76.2\%) were significantly related to death outcome, while delayed development of studied population show that $(21 \%)$ were significantly related to improved outcome and $(55.6 \%)$ were significantly related to recurrence outcome(Table 2). According to the past history of status epilepticus; Negative past history was significantly related to improved outcome $(84.2 \%)$, while the positive past history of status epilepticus of the studied population was significantly related to death outcome (23.8\%) (Table 2). 
SOHAG MEDICAL JOURNAL Clinico-laboratory profile in children with status epilepticus and correlation Vol. 24 No. 1 Jan 2020

Asmaa mansour

Table (2): Relation between immediate outcome and demographic and clinical features of the studied population

\begin{tabular}{|c|c|c|c|c|c|}
\hline Variable & $\begin{array}{c}\text { Improved } \\
\mathbf{N}=\mathbf{3 8}\end{array}$ & $\begin{array}{c}\text { Discharged with } \\
\text { neurological deficit } \\
\mathrm{N}=14\end{array}$ & $\begin{array}{c}\text { Recurrent status } \\
\mathrm{N}=\mathbf{2 7}\end{array}$ & $\begin{array}{c}\text { Died } \\
\mathrm{N}=21\end{array}$ & P-value \\
\hline $\begin{array}{c}\text { Age/months } \\
\text { Mean } \pm \text { SD } \\
\text { Median (range) } \\
\end{array}$ & $\begin{array}{c}44.6 \pm 52.7 \\
14.5(2-168) \\
\end{array}$ & $\begin{array}{c}51.0 \pm 39.8 \\
35.5(10-120) \\
\end{array}$ & $\begin{array}{c}32.0 \pm 33.3 \\
18(2.5-132) \\
\end{array}$ & $\begin{array}{c}24.3 \pm 41.5 \\
5(1.5-144)\end{array}$ & 0.02 \\
\hline \multicolumn{6}{|c|}{$\mathrm{P} 1=0.6, \mathrm{P} 2=0.96, \mathrm{P} 3=0.13, \mathrm{P} 4=0.16, \mathrm{P} 5=0.002, \mathrm{P} 6=0.05$} \\
\hline $\begin{array}{c}\text { Gender } \\
\text { F } \\
\text { M }\end{array}$ & $\begin{array}{l}17(44.7 \%) \\
21(55.3 \%)\end{array}$ & $\begin{array}{l}9(64.3 \%) \\
5(35.7 \%)\end{array}$ & $\begin{array}{c}8(29.6 \%) \\
19(70.4 \%)\end{array}$ & $\begin{array}{l}10(47.6 \%) \\
11(52.4 \%)\end{array}$ & 0.19 \\
\hline $\begin{array}{c}\text { Type of convulsion } \\
\text { Focal } \\
\text { GTCC } \\
\text { Mixed seizures type }\end{array}$ & $\begin{array}{c}6(15.8 \%) \\
26(68.4 \%) \\
6(15.8 \%) \\
\end{array}$ & $\begin{array}{l}4(28.6 \%) \\
6(42.9 \%) \\
4(28.6 \%) \\
\end{array}$ & $\begin{array}{c}7(25.9 \%) \\
14(51.9 \%) \\
6(22.2 \%) \\
\end{array}$ & $\begin{array}{c}11(52.4 \%) \\
2(9.5 \%) \\
8(38.1 \%) \\
\end{array}$ & 0.004 \\
\hline \multicolumn{6}{|c|}{$\mathrm{P} 1=0.24, \mathrm{P} 2=0.39, \mathrm{P} 3<0.0001, \mathrm{P} 4=85, \mathrm{P} 5=0.07, \mathrm{P} 6=0.008$} \\
\hline $\begin{array}{c}\text { GCS } \\
\text { Mean } \pm \text { SD } \\
\text { Median (range) }\end{array}$ & $\begin{array}{l}8.7 \pm 1.9 \\
8(5-13) \\
\end{array}$ & $\begin{array}{l}10.8 \pm 7.7 \\
10(7-15)\end{array}$ & $\begin{array}{l}10.9 \pm 2.9 \\
11(5-15)\end{array}$ & $\begin{array}{l}8.7 \pm 3.5 \\
7(5-15) \\
\end{array}$ & 0.002 \\
\hline \multicolumn{6}{|c|}{$P 1=0.09, P 2=0.009, P 3=1.00, P 4=1.00, P 5=0.17, P 6=0.03$} \\
\hline $\begin{array}{c}\text { Duration/minutes } \\
\text { Mean } \pm \text { SD } \\
\text { Median (range) }\end{array}$ & $\begin{array}{c}45.4 \pm 22.3 \\
35(30-120)\end{array}$ & $\begin{array}{c}53.8 \pm 19.3 \\
57.5(30-90)\end{array}$ & $\begin{array}{l}42.5 \pm 16.0 \\
34(30-90)\end{array}$ & $\begin{array}{l}52.8 \pm 14.0 \\
60(30-90)\end{array}$ & 0.02 \\
\hline \multicolumn{6}{|c|}{$P 1=0.08, P 2=0.61, P 3=0.02, P 4=0.04, P 5=0.82, P 6=0.01$} \\
\hline $\begin{array}{c}\text { Development } \\
\text { Normal } \\
\text { Delayed }\end{array}$ & $\begin{array}{c}30(79.0 \%) \\
8(21.0 \%)\end{array}$ & $\begin{array}{c}10(71.4 \%) \\
4(28.6 \%)\end{array}$ & $\begin{array}{l}12(44.4 \%) \\
15(55.6 \%)\end{array}$ & $\begin{array}{c}16(76.2 \%) \\
5(23.8 \%)\end{array}$ & 0.02 \\
\hline \multicolumn{6}{|c|}{$P 1=0.71, P 2=0.004, P 3=1.00, P 4=0.10, P 5=1.00, P 6=0.03$} \\
\hline $\begin{array}{c}\text { Past history of status } \\
\text { No } \\
\text { Yes }\end{array}$ & $\begin{array}{c}32(84.2 \%) \\
6(15.8 \%)\end{array}$ & $\begin{array}{c}14(100 \%) \\
0\end{array}$ & $\begin{array}{l}10(37.0 \%) \\
17(63.0 \%)\end{array}$ & $\begin{array}{c}16(76.2 \%) \\
5(23.8 \%)\end{array}$ & $<0.0001$ \\
\hline \multicolumn{6}{|c|}{$\mathrm{P} 1=0.17, \mathrm{P} 2<0.0001, \mathrm{P} 3=0.50, \mathrm{P} 4<0.0001, \mathrm{P} 5=0.07, \mathrm{P} 6=0.007$} \\
\hline $\begin{array}{c}\text { Family history of similar } \\
\text { condition } \\
\text { No } \\
\text { Yes } \\
\end{array}$ & $\begin{array}{c}32(84.2 \%) \\
6(15.8 \%)\end{array}$ & $\begin{array}{c}10(71.4 \%) \\
4(25.6 \%)\end{array}$ & $\begin{array}{c}21(77.8 \%) \\
6(22.2 \%) \\
\end{array}$ & $\begin{array}{c}19(90.5 \%) \\
2(9.5 \%)\end{array}$ & 0.50 \\
\hline $\begin{array}{l}\text { Consanguinity } \\
\text { Negative } \\
\text { positive }\end{array}$ & $\begin{array}{l}17(44.7 \%) \\
21(55.3 \%)\end{array}$ & $\begin{array}{l}5(35.7 \%) \\
9(64.3 \%)\end{array}$ & $\begin{array}{c}8(29.6 \%) \\
19(70.4 \%)\end{array}$ & $\begin{array}{c}3(14.3 \%) \\
18(85.7 \%)\end{array}$ & 0.12 \\
\hline
\end{tabular}

**pairwise comparison was made if the p-value was significant, p1 compared improved with neurological deficit, p2 compared improved with recurrent, $\mathrm{p} 3$ compared improved with died, p4 compared neurological deficit with recurrent, p5 compared neurological deficit with died, p6 compared recurrent with died and the p-value is significant when $<0.05$

The correlation between immediate outcome and laboratory investigation of the included population revealed that normal levels of serum ammonia of the examined patients were significantly related to improved outcome $(92.1 \%)$, while higher levels were significantly related to death outcome (38\%) (Table 3). Normal levels of serum lactate were significantly related to improved outcomes $(94.7 \%)$, while higher levels were significantly related to death outcomes (33.3\%) (Table 3). 
SOHAG MEDICAL JOURNAL Clinico-laboratory profile in children with status epilepticus and correlation Vol. 24 No. 1 Jan 2020

Asmaa mansour

Table (3): Relation between immediate outcome and Lab investigations of the studied population

\begin{tabular}{|c|c|c|c|c|c|}
\hline Variable & $\begin{array}{l}\text { Improved } \\
\mathbf{N}=38\end{array}$ & $\begin{array}{c}\text { Discharged with } \\
\text { neurological deficit } \\
\mathrm{N}=14\end{array}$ & $\begin{array}{c}\text { Recurrent status } \\
\mathbf{N}=\mathbf{2 7}\end{array}$ & $\begin{array}{l}\text { Died } \\
\mathrm{N}=21\end{array}$ & P-value \\
\hline $\begin{array}{l}\text { WBCs } \\
\text { Mean } \pm \text { SD } \\
\text { Median (range) }\end{array}$ & $\begin{array}{c}13.5 \pm 7.6 \\
10.6(5-29)\end{array}$ & $\begin{array}{l}9.3 \pm 3.0 \\
8(6-14)\end{array}$ & $\begin{array}{c}11.6 \pm 5.9 \\
12.8(4.5-22.7)\end{array}$ & $\begin{array}{c}9.5 \pm .5 \\
8(6-17.5)\end{array}$ & 0.29 \\
\hline $\begin{array}{l}\text { HGB } \\
\text { Mean } \pm \text { SD } \\
\text { Median (range) }\end{array}$ & $\begin{array}{c}10.7 \pm 1.5 \\
10(7.9-13)\end{array}$ & $\begin{array}{c}10.9 \pm 1.9 \\
11(7.9-13)\end{array}$ & $\begin{array}{c}9.8 \pm 0.9 \\
10(8-11.5)\end{array}$ & $\begin{array}{c}9.8 \pm 1.5 \\
10(6.8-12)\end{array}$ & 0.06 \\
\hline $\begin{array}{l}\text { Platelets } \\
\text { Mean } \pm \text { SD } \\
\text { Median (range) } \\
\end{array}$ & $\begin{array}{c}249.1 \pm 128.8 \\
334(155-667) \\
\end{array}$ & $\begin{array}{c}376.6 \pm 135.6 \\
435(199-667) \\
\end{array}$ & $\begin{array}{r}276.3 \pm 119.6 \\
228(96-499)\end{array}$ & $\begin{array}{c}308.8 \pm 171.3 \\
235(129-596) \\
\end{array}$ & 0.08 \\
\hline $\begin{array}{l}\text { Na } \\
\text { Mean } \pm \text { SD } \\
\text { Median (range) }\end{array}$ & $\begin{array}{c}136.3 \pm 5.7 \\
135(126-148)\end{array}$ & $\begin{array}{c}134.1 \pm 1.8 \\
133(132-137)\end{array}$ & $\begin{array}{c}135.4 \pm 5.5 \\
135(127-146)\end{array}$ & $\begin{array}{c}136 \pm 5.8 \\
134(130-151)\end{array}$ & 0.61 \\
\hline $\begin{array}{l}\text { K } \\
\text { Mean } \pm \text { SD } \\
\text { Median (range) } \\
\end{array}$ & $\begin{array}{r}3.9 \pm 0.8 \\
4(2.8-5.5) \\
\end{array}$ & $\begin{array}{c}3.7 \pm 0.5 \\
3.8(2.8-4.7) \\
\end{array}$ & $\begin{array}{r}4.1 \pm 0.6 \\
4(3.5-6) \\
\end{array}$ & $\begin{array}{l}4.1 \pm 0.7 \\
4(3-5.3) \\
\end{array}$ & 0.20 \\
\hline $\begin{array}{l}\text { Ca } \\
\text { Mean } \pm \text { SD } \\
\text { Median (range) }\end{array}$ & $\begin{array}{c}1.2 \pm 0.19 \\
1.1(0.9-1.7)\end{array}$ & $\begin{array}{c}1.2 \pm 0.2 \\
1.2(0.9-1.5)\end{array}$ & $\begin{array}{c}1.3 \pm 0.26 \\
1.2(0.9-1.7)\end{array}$ & $\begin{array}{c}1.3 \pm 0.4 \\
1.3(0.6-1.8)\end{array}$ & 0.21 \\
\hline $\begin{array}{l}\text { S. creatinine } \\
\text { Mean } \pm \text { SD } \\
\text { Median (range) }\end{array}$ & $\begin{array}{c}1.7 \pm 3.6 \\
0.5(0.3-12.4)\end{array}$ & $\begin{array}{c}0.5 \pm 0.2 \\
0.5(0.2-0.9)\end{array}$ & $\begin{array}{c}0.6 \pm 0.2 \\
0.5(0.2-1.0)\end{array}$ & $\begin{array}{c}0.6 \pm 0.2 \\
0.7(0.2-1.0)\end{array}$ & 0.48 \\
\hline $\begin{array}{l}\text { S. ammonia } \\
\text { Normal } \\
\text { High }\end{array}$ & $\begin{array}{c}35(92.1 \%) \\
3(7.9 \%)\end{array}$ & $\begin{array}{c}13(92.9 \%) \\
1(7.1 \%)\end{array}$ & $\begin{array}{c}20(74.1 \%) \\
7(25.9 \%)\end{array}$ & $\begin{array}{c}13(61.9 \%) \\
8(38.1 \%)\end{array}$ & 0.02 \\
\hline \multicolumn{6}{|c|}{$P 1=1.00, P 2=0.07, P 3=0.01, P 4=0.23, P 5=0.06, P 6=0.53$} \\
\hline $\begin{array}{l}\text { S. lactate } \\
\text { Normal } \\
\text { High } \\
\end{array}$ & $\begin{array}{c}36(94.7 \%) \\
2(5.3 \%)\end{array}$ & $\begin{array}{c}14(100 \%) \\
0\end{array}$ & $\begin{array}{c}22(81.5 \%) \\
5(18.5 \%)\end{array}$ & $\begin{array}{c}14(66.7 \%) \\
7(33.3 \%)\end{array}$ & 0.009 \\
\hline \multicolumn{6}{|c|}{$\mathrm{P} 1=1.00, \mathrm{P} 2=0.12, \mathrm{P3}=0.007, \mathrm{P} 4=0.15, \mathrm{P} 5=0.03, \mathrm{P} 6=0.24$} \\
\hline $\begin{array}{l}\text { Atrial blood gases } \\
\text { Normal } \\
\text { Acidosis }\end{array}$ & $\begin{array}{c}34(89.5 \%) \\
4(10.5 \%)\end{array}$ & $\begin{array}{c}14(100 \%) \\
0\end{array}$ & $\begin{array}{c}26(96.3 \%) \\
1(3.7 \%)\end{array}$ & $\begin{array}{c}18(85.7 \%) \\
3(14.3 \%)\end{array}$ & 0.34 \\
\hline
\end{tabular}

***airwise comparison was made if the p-value was significant, p1 compared improved with neurological deficit, $\mathrm{p} 2$ compared improved with recurrent, $\mathrm{p} 3$ compared improved with died, $\mathrm{p} 4$ compared neurological deficit with recurrent, p5 compared neurological deficit with died, p6 compared recurrent with died.

The correlation between immediate outcome and the other investigations of the studied population revealed that normal examination of the studied lumbar puncture was significantly related to improved outcome $(55.3 \%)$, while each encephalitis and meningitis were significantly related to improved outcome $(18.4 \%),(21 \%)$ in order, patients who did not have lumbar puncture(failure) were significantly related to death outcome $(28.6 \%)$ (Table 4$)$.
Normal electroencephalography findings were significantly related to improved outcomes $(84.2 \%)$ while epileptic discharges findings were significantly related to recurrence outcome (44.4\%) (Table 4). Normal findings of brain imaging (CT/MRI brain) of the included patients were significantly related to improved outcomes (57.9\%), while abnormal findings were significantly related to death outcome $(90.5 \%)$ (Table 4). 
SOHAG MEDICAL JOURNAL Clinico-laboratory profile in children with status epilepticus and correlation

Table (4): Relation between immediate outcome and other investigations of the studied population.

\begin{tabular}{|c|c|c|c|c|c|}
\hline Variable & $\begin{array}{l}\text { Improved } \\
\mathbf{N}=38\end{array}$ & $\begin{array}{c}\text { Discharged with } \\
\text { neurological deficit } \\
\mathrm{N}=14 \\
\end{array}$ & $\begin{array}{c}\text { Recurrent status } \\
\mathbf{N}=\mathbf{2 7}\end{array}$ & $\begin{array}{l}\text { Died } \\
\mathrm{N}=\mathbf{2 1}\end{array}$ & P-value \\
\hline $\begin{array}{l}\text { EMS } \\
\text { Normal } \\
\text { Abnormal }\end{array}$ & $\begin{array}{c}38(100 \%) \\
0\end{array}$ & $\begin{array}{c}14(100 \%) \\
0\end{array}$ & $\begin{array}{c}26(96.3 \%) \\
1(3.7 \%)\end{array}$ & $\begin{array}{c}20(95.2 \%) \\
1(4.8 \%)\end{array}$ & 0.52 \\
\hline $\begin{array}{l}\text { C.S.F analysis } \\
\text { Normal } \\
\text { Encephalitis } \\
\text { Meningitis } \\
\text { failed }\end{array}$ & $\begin{array}{c}21(55.3 \%) \\
7(18.4 \%) \\
8(21.0 \%) \\
2(5.3 \%)\end{array}$ & $\begin{array}{c}13(92.9 \%) \\
1(7.1 \%) \\
0 \\
0\end{array}$ & $\begin{array}{c}19(70.4 \%) \\
2(7.4 \%) \\
0 \\
6(22.2 \%)\end{array}$ & $\begin{array}{c}15(71.4 \%) \\
0 \\
0 \\
6(28.6 \%)\end{array}$ & 0.001 \\
\hline \multicolumn{6}{|c|}{$P 1=0.08, P 2=0.01, P 3=0.003, P 4=0.16, P 5=0.05, P 6=0.42$} \\
\hline $\begin{array}{l}\text { EEG } \\
\text { Normal } \\
\text { Epileptic discharges } \\
\text { Hippes arrhythmia }\end{array}$ & $\begin{array}{c}32(84.2 \%) \\
6(15.8 \%) \\
0 \\
\end{array}$ & $\begin{array}{c}8(57.1 \%) \\
6(42.9 \%) \\
0 \\
\end{array}$ & $\begin{array}{c}13(48.2 \%) \\
12(44.4 \%) \\
2(7.4 \%) \\
\end{array}$ & $\begin{array}{c}21(100 \%) \\
0 \\
0 \\
\end{array}$ & 0.001 \\
\hline \multicolumn{6}{|c|}{$\mathrm{P} 1=0.04, \mathrm{P} 2=0.005, \mathrm{P} 3=0.06, \mathrm{P} 4=0.55, \mathrm{P} 5=0.001, \mathrm{P} 6<0.0001$} \\
\hline $\begin{array}{l}\text { MRIICT brain } \\
\text { Normal } \\
\text { Abnormal } \\
\end{array}$ & $\begin{array}{l}22(57.9 \%) \\
16(42.1 \%)\end{array}$ & $\begin{array}{c}4(28.6 \%) \\
10(71.4 \%)\end{array}$ & $\begin{array}{c}6(22.2 \%) \\
21(77.9 \%) \\
\end{array}$ & $\begin{array}{c}2(9.5 \%) \\
19(90.5 \%)\end{array}$ & 0.001 \\
\hline
\end{tabular}

**pairwise comparison was made if the p-value was significant, p1 compared improved with neurological deficit, p2 compared improved with recurrent, p3 compared improved with died, p4 compared neurological deficit with recurrent, p5 compared neurological deficit with died, p6 compared recurrent with died and the p-value is significant when $<0.05$.

The correlation between immediate outcome and the medical treatment during the attack of $\mathrm{SE}$ of the included population revealed that patients who were treated with levetiracetam as first-line treatment were significantly related to improved outcome $(65.8 \%)$ and to death outcome (19\%), while patients who were treated with midazolam as the first line of treatment were significantly related to improved outcome $(29 \%)$ and death outcome $(38.1 \%)$, finally, patients treated with combination of both drugs were significantly related to death outcome (42.9\%) (Table 5).

Table (5): Relation between immediate outcome and Medical treatment during an attack of the studied population

\begin{tabular}{|c|c|c|c|c|c|}
\hline Variable & $\begin{array}{l}\text { Improved } \\
\mathbf{N}=\mathbf{3 8}\end{array}$ & $\begin{array}{c}\text { Discharged with } \\
\text { neurological } \\
\text { deficit } \\
\mathrm{N}=14\end{array}$ & $\begin{array}{c}\text { Recurrent status } \\
\mathbf{N}=\mathbf{2 7}\end{array}$ & $\begin{array}{c}\text { Died } \\
\mathbf{N}=21\end{array}$ & P-value \\
\hline $\begin{array}{l}\text { Midazolam } \\
\text { Levetiracetam } \\
\text { Midazolam \& } \\
\text { levetiracetam } \\
\text { Other antiepileptic drugs }\end{array}$ & $\begin{array}{c}11(29.0 \%) \\
25(65.8 \%) \\
0 \\
2(5.2 \%)\end{array}$ & $\begin{array}{c}3(21.4 \%) \\
9(64.3 \%) \\
2(14.3 \%) \\
0\end{array}$ & $\begin{array}{c}2(7.4 \%) \\
16(59.3 \%) \\
9(33.3 \%) \\
0\end{array}$ & $\begin{array}{c}8(38.1 \%) \\
4(19.0 \%) \\
9(42.9 \%) \\
0\end{array}$ & $<0.0001$ \\
\hline
\end{tabular}

**pairwise comparison was made if the p-value was significant, $\mathrm{p} 1$ compared improved with neurological deficit, $\mathrm{p} 2$ compared improved with recurrent, p3 compared improved with died, p4 compared neurological deficit with recurrent, p5 compared neurological deficit with died, p6 compared recurrent with died and the p-value is significant when $<0.05$.

The correlation between immediate outcome and possible etiology of the studied population revealed that patients presented with hypoxicischemic encephalopathy were significantly related to death outcome $(38.1 \%)$, while patients presented with epilepsy were significantly related to recurrence outcome
$(59.3 \%)$ and to the improved outcome $(15.8 \%)$. Patients presented with basal ganglia infarction were significantly related to death outcome $(28.6 \%)$, finally, patients presented with SE after trauma were significantly related to had neurological deficit $(28.6 \%)$ (Table 6). 
SOHAG MEDICAL JOURNAL Clinico-laboratory profile in children with status epilepticus and correlation

Vol. 24 No. 1 Jan 2020

Asmaa mansour

Table (6): Relation between immediate outcome and possible etiology of the studied population

\begin{tabular}{|c|c|c|c|c|c|}
\hline Variable & $\begin{array}{c}\text { Improved } \\
\mathbf{N}=38\end{array}$ & $\begin{array}{c}\text { Discharged with } \\
\text { neurological } \\
\text { deficit } \\
\mathrm{N}=14 \\
\end{array}$ & $\begin{array}{c}\text { Recurrent } \\
\text { status } \\
\mathbf{N}=27\end{array}$ & $\begin{array}{c}\text { Died } \\
\mathrm{N}=21\end{array}$ & P-value \\
\hline Acute disseminated encephalomyelitis & $5(13.2 \%)$ & $1(7.1 \%)$ & $\mathbf{0}$ & $\mathbf{0}$ & 0.09 \\
\hline Hypoxic ischemic encephalopathy & $2(5.3 \%)$ & $2(14.3 \%)$ & $2(7.4 \%)$ & $8(38.1 \%)$ & 0.004 \\
\hline \multicolumn{6}{|c|}{$P 1=0.29, P 2=1.00, P 3=0.002, P 4=0.60, P 5=0.25, P 6=0.01$} \\
\hline Acute necrotizing encephalopathy & $2(5.3 \%)$ & $\mathbf{0}$ & $\mathbf{0}$ & $\mathbf{0}$ & 0.34 \\
\hline Basal ganglia infarction & $\mathbf{0}$ & $\mathbf{0}$ & $\mathbf{0}$ & $6(28.6 \%)$ & $<0.0001$ \\
\hline \multicolumn{6}{|c|}{$P 1=1.00, P 2=1.00, P 3=0.001, P 4=1.00, P 5=0.06, P 6=0.004$} \\
\hline Encephalitis & $5(13.2 \%)$ & $1(7.1 \%)$ & $2(7.4 \%)$ & $\mathbf{0}$ & 0.36 \\
\hline Epilepsy & $6(15.8 \%)$ & $6(42.9 \%)$ & $16(59.3 \%)$ & $\mathbf{0}$ & $<0.001$ \\
\hline \multicolumn{6}{|c|}{$P 1=0.06, P 2<0.0001, P 3=0.08, P 4=0.32, P 5=0.002, P 6<0.0001$} \\
\hline Hypertensive encephalopathy (CRF) & $4(10.5 \%)$ & $\mathbf{0}$ & $\mathbf{0}$ & $\mathbf{0}$ & 0.08 \\
\hline Intracranial hemorrhage & $2(5.3 \%)$ & $\mathbf{0}$ & $\mathbf{0}$ & $2(9.5 \%)$ & 0.32 \\
\hline Meningitis & $8(21.1 \%)$ & $\mathbf{0}$ & $\mathbf{0}$ & $\mathbf{0}$ & 0.003 \\
\hline \multicolumn{6}{|c|}{$P 1=0.09, P 2=0.02, P 3=0.04, P 4=1.00, P 5=1.00, P 6=1.00$} \\
\hline Post traumatic status & $\mathbf{0}$ & $4(28.6 \%)$ & $\mathbf{0}$ & $\mathbf{0}$ & $<0.0001$ \\
\hline \multicolumn{6}{|c|}{$P 1=0.001, P 2=1.00, P 3=1.00, P 4=0.003, P 5=0.009, P 6=1.00$} \\
\hline Propionic acidemia (metabolic cause) & $\mathbf{0}$ & $\mathbf{0}$ & $1(3.7 \%)$ & $14.8(\%)$ & 0.52 \\
\hline Hypoglycemia due to insulin overdose & $2(5.3 \%)$ & $\mathbf{0}$ & $\mathbf{0}$ & $\mathbf{0}$ & 0.34 \\
\hline White matter disease & $2(5.3 \%)$ & $\mathbf{0}$ & $4(14.8 \%)$ & $2(9.5 \%)$ & 0.34 \\
\hline Not confirmed etiology & $\mathbf{0}$ & $\mathbf{0}$ & $2(7.4 \%)$ & $2(9.5 \%)$ & 0.20 \\
\hline
\end{tabular}

**pairwise comparison was made if the p-value was significant, p1 compared improved with neurological deficit, p2 compared improved with recurrent, p3 compared improved with died, p4 compared neurological deficit with recurrent, p5 compared neurological deficit with died, p6 compared recurrent with died and the p-value is significant when $<0.05$.

\section{Discussion}

In the present study, the sex distribution shows a slightly higher incidence of status epilepticus in males than females. many other studies have done both in pediatrics and adults shows a similar type of results Gulati et al. [8], observed $22(70 \%)$ patients out of 31 were male. the same fact was also found in Chen $e t$ al. [9] study.

It was observed that GTCS type of seizure is the commonest of all 3 types (48\%) in our study, the similar incidence was observed by Kwong et al. [10], Gulati et al., [8], $(54 \%)(63.3 \%)$ in order were GTCS.

The same results of Arun et al. [7], agree that $66 \%$ were a generalized tonic-clonic seizure. Adhikari et al. [11], study and Chen et al. [9], observe the same result.

After GTCS type of seizure; focal seizure is the commonest type which came to $28 \%$.
Gulati et al. [8], observed $26.6 \%$ were focal and Arun et al. [7], observed focal seizure were $18 \%$.

Out of 100 children in our study (72\%) never had the previous episode of seizure and (28\%)

had the previous history of seizure and the same result was found by Arun et al. [7] as Out of 50 children (72\%) never had previous episode of seizure and (28\%) had the previous history of seizure.

Garzon et al. [12], found that $59.4 \%$ were known epileptic and $40.5 \%$ were not epileptic. Gulati et al. [8], observed that $53.3 \%$ are epileptic, In our study, we found that $28 \%$ are epileptic.

In the present study, $18 \%$ had a family history of seizure and $82 \%$ did not have any family history of seizure, this agreement with the study of Arun et al. [7], as 26\% had a family 
SOHAG MEDICAL JOURNAL Clinico-laboratory profile in children with status epilepticus and correlation

history of seizure and $74 \%$ did not have any family history of seizure.

In the present study, $32 \%$ of children had mild to severe forms of developmental delay and $82 \%$ of children had normal milestones. In a study done by Gulati et al. [8], 25\% had developmental delays.in the study of Arun $\boldsymbol{e t}$ al. [7], 26\% of children had mild to severe forms of developmental delay and $74 \%$ of children had normal milestones.

In the current study, there was a total of $21 \%$ mortality, in a study done by Gulati et al. [8], Kwong et al. [10], Arun et al. [7], had a mortality of $30 \%, 11 \%, 4 \%$ in order.

In the current study, $38 \%$ of the total children were discharged without any neurological deficit, $14 \%$ discharged with neurological deficit and $27 \%$ recure again with status epilepticus but in Arun et al. [7]study, 94\% of the total children were discharged without any neurological deficit.

In the current study, (28\%) had epilepsy, following that hypoxic encephalopathy was the commonest (14\%). Encephalitis and Meningitis and white matter disease each had $8 \%$. $(2 \%)$ of children presented with status due to propionic academia.

(4\%) of children come with unconfirmed etiology.

In Arun et al. [7] (56\%) had epilepsy, following that febrile status was the commonest (24\%). SOL was $6 \%$. Encephalitis and Meningitis each had 4\%; $1 \%$ had Vasculitis, Hypoxic encephalopathy, and neem oil poisoning.

Our findings were contrary to Chen et al. [9] study where febrile seizures were the commonest causes followed by trauma, epilepsy and CNS infections.

Singh and Suryavanshi [13] study observed that CNS infections to be the commonest cause of seizure in the pediatric age group, followed by SOL, epilepsy, febrile seizures, and metabolic causes.

In our study Lumbar puncture was done to 86 patients out of which 68 were normal; 18patients had CSF c/s positive and 14 patient in whom CSF not done for them, in study of Arun et al. [7], Lumbar puncture was done to 19 patients out of which 15 were normal; 2 patients had CSF c/s positive and 2 had HSV positive.

Neuroimaging was done to 100 patients out of which 34 were normal and 66 patients had abnormal Neuroimaging, in the study of Arun et al. [7], Neuroimaging was done to 15 patients out of whom 10 were normal. 3 patients had space-occupying lesions; 1 patient had congenital anomaly and 1 had ischemia of cerebral arteries.

EEG was done in100patients out of which 24patient had seizure activity in our study while in Arun et al. [7]study EEG was done in 19 patients out of which 16 patients had seizure activity.

In our study we found that $38 \%$ of patients were improved without any neurological deficits and patients who had epileptic seizures were related significantly to improved outcome $15.8 \%$ of the totally improved patients while recurrence outcome represents about $59.3 \%$ in those patients who presented with epilepsy. patients presented with hypoxic-ischemic encephalopathy show the highest mortality rate $(38.1 \%)$ among the deaths $(21 \%)$.

Singh and Suryavanshi [13] study show that febrile seizures had the best outcome with all patients discharged without any deficits; CNS infections need to be focused more because of the highest morbidity and mortality. Although the immediate outcome in SOL and epilepsy patients seems good in terms of discharge (93\% and $84 \%$ respectively).

Many Egyptian studies had compared outcomes of status epilepticus such asShehata HS et al. [14] study found that at least onethird of tuberous sclerosis patients had intractable seizures refractory to both medical and surgical treatment, these patients are at particular risk of developing SE which is the chief cause of death among tuberous sclerosis patients.

\section{Conclusion:}

In this study, we found that in the included children, epilepsy is the most common cause of status epilpticus and those epileptic children were significantly related to the improvement and recurrence outcome. patients presented with hypoxic-ischemic encephalopathy were 
significantly related to death outcome and all patients presented with status epilepticus should have a full clinical examination and laboratory investigations.

\section{Limitation of work:}

- It is a single centered study, so the results cannot be generalized \& we need multicenter studies for assessment of the clinical and the laboratory profiles of status epilepticus and the immediate outcome of the patients.

- Relatively small sample size.

\section{Recommendation:}

- Patients presented with status epilepticus should be subjected to a detailed history and complete general, systemic and neurological examination on admission.

- Patients also should be subjected to detailed laboratory investigations, EEG and brain imaging.

- Rapid initiation of antiepileptic drugs helps to improve the outcome of SE.

\section{References}

1. DeLorenzo RJ, Hauser WA, Towne AR et al. (1996): A prospective, population-based epidemiologic study of status epilepticus in Richmond, Virginia. Neurology, 46: 10291035.

2. Khurana DS (2000): Treatment of status epilepticus. Lnd J Pediatr., 67: 1.

3. International League Against Epilepsy (1989): Proposal for classification of the epilepsies and epileptic syndromes. Epilepsia., 30: 389-399.

4. Hanhan UA, Fiallos MK, Orlowski JP (2001):. Status epilepticus. Pediatr Clin North Am., 48:683- 94.

5. Berg AT, Shinnar S, Testa FM, Levy SR, Frobish D, Smith SN (2004): Status epilepticus after the initial diagnosis of epilepsy in children. Neurology, 63: 1027-34.

6. Segeleon JE, Haun SE (2000): Status epilepticus. In: Tobias JD, editors. Pediatric Critical Care: The Essentials. Chennai: Orient Longman, Pp. 207-21.

7. Arun PT, Shruthi TK, Rajakumar PS, Shuba S (2017): Profile on status epilepticus, clinical features and lab parameters and outcome in pediatric intensive care. Int $\mathrm{J}$ Contemp Pediatr., 4(4):1310-1316.

8. Gulati S, Kalra V, Sridhar MR (2005): Status Epilepticus in Indian Children in a tertiary care center. Indian J Pediatruics., 72:105-8.

9. Chen CY, Chang YJ, Wu HP (2010): New-onset seizures in a pediatric emergency. Pediatr Neonatol., 51:103-11.

10. Kwong KL, Lee SL, Yung A, Wong VC (1995): A study of the etiology and short term outcome of status epilepticus in children. J Child Health, (5):395-8.

11. Adhikari S, Sathian B, Koirala DP, Rao KS (2013): Profile of children admitted with seizures in a tertiary care hospital of Western Nepal. BMC Pediatr., 13:43-46.

12. Garzon E, Fernandes RM, Sakamoto AC (2003): Analysis of clinical characteristics and risk factors for mortality in human status epilepticus. Seizure, 12(6):337-45.

13. Singh RD, Suryavanshi S (2016): A hospital-based study on clinicoetiological profile of seizures in children - a Kanpur (U.P., India) experience. International Journal of Contemporary Medical Research, 3(10):3003-3007.

14. Shehata HS, AbdelGhaffar HM, NasreldinM et al. (2017): clinical patterns and outcomes of status epilepticus in patients with tuberous sclerosis complex.Dove press.,13:779785. 
SOHAG MEDICAL JOURNAL Clinico-laboratory profile in children with status epilepticus and correlation Vol. 24 No. 1 Jan 2020 